\title{
Index
}

Aaron, Jane 282

Aboriginal 10, 83, 92, 133, 146, 228-31, 285-6, 302 see also 'First Nations'

Achebe, Chinua 12-13, 110, 150, 165-6, 172, 293

Anthills of the Savannah 123, 152-9

Things Fall Apart 100, 152

Africa 7, 11, 17, 26, 34, 91-3, 96-7, 99-100, 115, 122, 143, 149, 165, 200, 202, 226, 236, 245, 265, 280-1, 291, 299-300

Ahluwalia, Pal 47, 55

Ahmad, Aijaz 34, 58, 147-8, 256

Ahmad, Dohra 148

Algeria 22, 67, 101

Ali, Monica 238

Althusser, Louis 44-5

ambivalence $65-8,75,139,142$, 205-6, 251

Anderson, Benedict 82, 86-8, 114, 123

Anthias, Floya 132, 137, 139, 159

anti-colonial nationalism; see nationalism

Antigua 163, 170-2

Appadurai, Arjun 83, 124, 305-6, 308

Appiah, Kwame Anthony 296, 310, 312

Ashcroft, Bill 29-33, 35-7, 47, 55, 145-9

see also Empire Writes Back, The
Asia 7, 34, 299-300

Augé, Marc 303-4

Austen, Jane 17

Mansfield Park 27, 169, 171-2

Australasia 7, 200, 301

Australia 3, 10-11, 15, 17, 110, 137, 144-5, 169, 228-9, 236, 280, 285, 301, 303

'Mabo Case' and 92

terra nullius and 92

Azim, Firdous 183

Baldwin, James 268

Balibar, Etienne 124, 133-4

'Baptist War' (1831-2) 183

Barbados 17, 235

Beckett, Samuel 282

Belsey, Catherine 199

Berlin Conference (1884-85) 91

Bhabha, Homi K 27-8, 32, 34-5, 48, 61-70, 75-6, 139-41, 143, 157, 205-6, 238, 251-7, 259-60, 263-4, 287, 294, 297, 300 see also ambivalence; 'in-between'; mimicry

Biafra 134

'Black Atlantic'; see Paul Gilroy

Boehmer, Elleke 10, 159, 197, 281, 286

Brah, Avtar 238, 241, 260-1

Brantlinger, Patrick 181-2

Brathwaite, Edward Kamau 150-2 
see also 'nation language'

Brennan, Timothy 82, 84

British Empire 7, 10-11, 13, 144, 169, 234-5, 281, 286

see also Empire

British Overseas Territories 7

Brittan, Alice 92

Brontë, Charlotte

Jane Eyre 27, 162, 169, 172-194, 209

Bryce-Okunlola, Jane 226

Cabral, Amilcar 90, 93, 302

Canada 7, 10-11, 69, 92, 133, 146-7, 280, 284, 291, 301

see also 'First Nations'

capitalism 8-10, 80, 224, 294-8, 302, $307-8$

Carby, Hazel 203, 207-9

Caribbean 7-8, 11-12, 15, 17, 93, 96-7, 100, 103, 122, 150-2, 167, 170-1, 183, 185-6, 188, 191, 236, 240, 245, 258, 265, 267, 269, 271-2, 280-2, 291, 299

Carr, Helen 186, 193

Castle, Gregory 281

Castro, Fidel 299

Césaire, Aimé 22, 93-4, 96, 98-100, 167,282

Notebook of a Return to My Native Land 97

Chatterjee, Partha 125-9

Chew, Shirley 19

Chicano/a 283

Childs, Peter 9, 33

China 11, 34, 236, 249

Chow, Rey 238

Chrisman, Laura 58

class 31, 67, 156, 214, 238-9, 255, 261, 264, 296

Clifford, James 39, 236, 249, 266

Coetzee, J. M. 293, 311

Foe 36

Cohen, Robin 237

colonial discourses 19-21, 26-8, 31,
$35,38,40-1,44-7,57,60-2,64$, $67-70,73,77,80,94-5,98-101$, 109-10, 139, 165, 169, 177-8, 180-1, 184, 189, 201-2, 205, 209, $223,262,277,279,313$

colonialism definition of 8-11

Columbus, Christopher 8

Commonwealth literature 12-19, 26, 28, 32, 280-1, 284-5, 289

nation and 15

Commonwealth of Nations 13, 15

Connell, John 242

Conrad, Joseph 166

Heart of Darkness 26, 165, 172

contrapuntal 171

cosmopolitanism 309-10

Cuba 299

cultural studies 37

Dabydeen, David 262-3

D'Aguiar, Fred 238

Dangarembga, Tsitsi 293

Davies, Carole Boyce 39, 136, 226-7, 230

decolonisation 7-8, 10-12, 39, 48, 80, $93,110,119,122,126,134$, $136-7,206,278$

diaspora and 235-6

Deleuze, Gilles 218-19

Derrida, Jacques 27, 209, 227, 300

Desai, Anita 147

diaspora definition of 236-7

Dirlik, Arif 62, 294-5, 297, 299

Dominica 185

dominions 10-11, 13, 286

Donaldson, Laura 208

'double colonisation' 200-2, 206-7, 229

During, Simon 286

Eagleton, Terry 294

Egypt 24, 214

Eliot, George 17 


\section{Middlemarch 87}

Emecheta, Buchi 238

Joys of Motherhood, The 203

Empire 7-8, 11-13, 21, 25, 39-40, 45, 50-1, 59, 69-70, 73-4, 76, 80,

$110,170,186,284,298-9,300$

see also British Empire

globalisation as 308-9

Empire Writes Back, The 28-32, 146

Engels, Friedrich 34

Equiano, Olaudah 235

ethnicity $133,135,137-8,141,244$, $248,261,264,266$

definition of 132

Fanon, Frantz 24-6, 28, 44-5, 103, 112-18, 120, 128-9, 143, 300, 313 see also national consciousness Black Skin, White Masks 22-3, 62, 66,271

national culture and 93, 101-2, 104-7, 109-10, 114, 127

native intellectual and $102-7$, 114-16

Wretched of the Earth, The 22, 102, 107

Featherstone, Simon 37-8, 282, 286 feminism 3, 26, 197, 207-8, 213-14, 216, 224, 226-7

definition of 198-9

see also 'First World' feminism

Fiji 14-15

'First Nations' 10, 31, 133, 302 see also Aboriginal

'First World' feminism 198-200, 206-7, 209, 211, 213, 224-5

Forsdick, Charles 283

Forster, E. M.

A Passage to India 202

Foucault, Michel 24, 27, 45-6, 62, $130,209,218-19,300$

Frame, Janet 12

Francophone postcolonial studies 283

Fraser, Robert 311

Freud, Sigmund 51, 254, 287
Fryer, Peter 234

Furphy, Joseph 110

Gandhi, Leela 33

Gandhi, Mahatma 128, 205, 302

Garan Kouyaté, Tiémoho 302

Gellner, Ernest 81

gender 31, 67-8, 124, 152, 156-7, 168, 197, 199, 203-6, 215, 220, 256, 261,281

diaspora and 238-9, 244

nationalism and 111, 118, 135-8, 155

Negritude and 100

Orientalism and 53-4, 59

'race' and 207-8

generational differences 237-8, 246

George, Rosemary Marangoly 131

Ghana 11

Ghosh, Amitav 147, 238

Gikandi, Simon 122, 124

Gilroy, Beryl

Boy-Sandwich 240, 269-273

Gilroy, Paul 90, 122, 125-6, 132, 238-9, 250, 257, 297, 310, 312

'Black Atlantic' and 249, 264-8, 271, 282

'planetary humanism' and 310, 312

globalisation 9, 239, 297, 306-313 passim definition of 304-5

Goebel, Walter 265

Gramsci, Antonio 24, 58, 130

Griffiths, Gareth 29-33, 290, 292 see also Empire Writes Back, The

Guevara, Che 299, 302

Guha, Ranajit 130-1, 155, 219, 226

Guinea-Bissau 90

Gunesekera, Romesh 238

Guyana 269

Hall, Stuart 38, 238, 261, 297

'New Ethnicities' 257-60, 264

Hannan, June 198

Hardt, Michael 123, 307-9, 312 
Harrison, Nicholas 26, 166, 168, 283

Head, Bessie 226

Heaney, Seamus 282

Hobsbawm, Eric 82

Hodge, Bob 32, 92

'Holy Trinity' 27, 33

home 85 , 89, 238, 241-50, 254-5, 266, 270

Hong Kong 11, 15

Huggan, Graham 37-8, 276

postcolonial exotic and $311-12$

Hulme, Peter 167

hybridity $240,253-7,260-2,264,290$, 294-5, 309

imperialism 8-10, 108, 122, 170, 176, $178,183,235,313$

globalisation as 307

'in-between' 245, 247-52, 306

indentured labourers 8, 240-1

India 3, 11-12, 15, 27, 65-7, 69-72,

$127,147-8,162,164,169,174-5$, $185,205,214,220,230,240-1$, $243,256,280,285,287$

Innes, C. L. 110-11

internal colonialism 39, 147

interpellation 44

Iran 214

Ireland 3, 7, 110, 280-2, 286

Northern Ireland and 11

Jamaica 3, 11, 174-6, 179-80, 182, $185-7$

James, C. L. R. 300

JanMohamed, Abdul 180

Jeffares, A. Norman 14, 16, 18

Joyce, James 282

Judd, Denis 8

Kabbani, Rana 50, 201-2

Katrak, Ketu H. 205

Keating, Peter 70, 75

Kenya 3, 117-19, 129, 135, 150

'Mau Mau' rebellion and 112

Kenyatta, Jomo 112
Keown, Michelle 236

Kincaid, Jamaica 163

King, Bruce 122

King, Russell 242

Kingsley, Mary

Travels in West Africa 202

Kipling, Rudyard 247

Departmental Ditties 70

The fungle Book 247

'The Overland Mail' 47, 69-77, 169

Kristeva, Julia 212, 217, 226

Kureishi, Hanif 238, 246-51, 253, 268

Lacan, Jacques 27, 62, 254, 287

Lahiri, Jhumpa 238

Lamming, George 167 Season of Adventure 17

LaRocque, Emma 146-7

Latin America 34, 299-300

Lawrence, T. E. Seven Pillars of Wisdom, The 61

Lawson, Alan 44-5

Lawson, Henry 80

Lazarus, Neil 33, 126-7, 296

'liberal humanist' 17

Lloyd, David 282

Loomba, Ania 33, 44

Macaulay, Thomas (Lord) 66, 164

McClintock, Anne 31, 205, 277-8

MacKenzie, John 57, 73

McLeod, A. L. 15

McLeod, John 33

Madsen, Deborah L. 283

Madmoman in the Attic, The 176, 209

Malaysia 280

Malta 15

manichean 180

Martinique 22, 94, 96-7

Marx, Karl 34, 130, 227, 279

Marxism 3, 227, 295-6, 299-300, 302

Mercer, Kobena 259

Merolla, Daniela 234

Meyer, Susan 175, 183

'Middle Passage' 265-6 
migrancy 237-9, 242, 244, 250, 281 see also diaspora

Mills, Sara 59-60, 68, 202, 205

mimicry 65, 67-8, 206

Mishra, Sudesh 238

Mishra, Vijay 32, 92

modernity 9, 84, 125-6

'Black Atlantic' and 265

Mohanty, Chandra Talpade 213-17, 225,227

Moore, Jane 199

Moore-Gilbert, Bart 58, 222

Morgan, Sally My Place 200, 228-32

Morris, Mervyn 84-5

Morrison, Toni Beloved 255

Morton, Stephen 209, 221, 223

Mukherjee, Arun P. 31, 288-92

Mukherjee, Bharati 238

Mukherjee, Meenakshi, 162-3, 165, 287-8

Murphy, David 236, 283

Naipaul, V. S. 12, 240-1, 246, 266 The Mimic Men 67

Narayan, R. K. 12, 147 The Guide 288

Nasta, Susheila 204, 225-6, 239

nation myth of $81-6,88-90,94,114$, $123-4,134$

national consciousness 93, 101-2, 104-7, 109-14, 118-19, 127, 149

see also Fanon, Frantz

nationalism, nationalisms anti-colonial and 11, 80-1, 90-1, 93, 125-30, 136, 138, 296

'nation language' 150-2

Negri, Antonio 123, 307-9, 311

Negritude 93-100, 102-3, 106, 109, $132,143,282$

neo-colonial 31, 117, 148, 278, 288-9, 291, 293-4, 304 neo-colonialism 46, 108, 112, 278-9, 292

Neruda, Pablo 282

New Caledonia 284

Newman, Judie 175, 191

New Zealand 3, 10-12, 69, 92, 280, 301

Maori and 11, 31, 299, 301

Ngugi wa Thiong'o 21, 25-6, 148-51, 206, 257

A Grain of Wheat 81, 107, 111-20, $128-9,135,158$

Nigeria 3, 11-12, 14, 91, 134-5, 152-3, 203, 235

Njau, Rebeka 226

Nkrumah, Kwame 299-300

Nwapa, Flora 226

Nyman, Jopi 293

Oriental, Orientalism see Said, Edward W.

Padmore, George 300

Pakistan 11, 214, 243, 246, 248

Parry, Benita 9, 70, 143, 222, 284, 293, 309

Paterson, A. B. 'Banjo' 80

patriarchy definition of 199

Petersen, Kirsten Holst 201, 204

Philippines 280

Phillips, Caryl 238, 245, 248, 264, 270

Ponzanesi, Sandra 234

Porter, Dennis 56, 58, 61

postcolonial exotic see Huggan,

Graham

poststructuralism 3, 218, 251

Pratt, Mary Louise 202

Procter, James 236

$$
\begin{aligned}
& \text { 'race' } 98-9,133,141,168,177,230, \\
& 238-9,244,248,258,261,264, \\
& \quad 266,270 \\
& \text { definition of 131-2 } \\
& \text { Radhakrishnan, R. } 262
\end{aligned}
$$


Rhys, Jean

Wide Sargasso Sea 162, 167, 185-94

Richards, David 49

Rushdie, Salman 25, 29, 71, 147, 242-3, 245-6, 248-51, 253, 293 , 310

Rutherford, Anna 201, 204

Sahgal, Nayantara 147

Said, Edward W. 25, 27, 35, 62, 65, 67-8, 71-2, 75, 170-1, 181, 294, 297

see also contrapuntal

Culture and Imperialism 32, 60, 169, $172,282,286$

Orientalism 24, 26, 32, 34, 46-61, $63,73,205,300$

Sancho, Ignatius 235

Saro-Wiwa, Ken 14

Sartre, Jean-Paul 22

sati 185,220

Saudi Arabia 214

Schabio, Saskia 265

Schoene, Berthold 282

Scotland 282

Scott, David 297

Second World War 11, 13, 85, 122, 199, 228, 235

Selvon, Sam 19-22, 38

Senegal 94

Senghor, Léopold 93-6, 98-100, 110, 302

settlement $8-10,12,24,63,108,301$, 307

settler 31, 92, 285-6, 301

'settler' nations 10

language in 144-7, 149

sexuality 136-7, 214

Shackleton, Mark 237

Shakespeare, William 167, 183

Macbeth 182

Tempest, The 166

Shohat, Ella 278-9, 285-6

Singapore 280
'Singapore Declaration of Commonwealth Principles' (1971) 13

Sivanandan, Tamara 90

slaves 8, 150, 185, 234, 267

slave trade 58,183

Smith, Anna Marie 235

Smith, Zadie 238

South Africa 10-11, 69, 83, 137, 256, $281,301,311$

South Asia 11, 91-2, 122, 200, 226, 236, 246, 250, 280-1

Soyinka, Wole 110

Spivak, Gayatri Chakravorty 28, 34-5, 48, 175, 200, 209, 211-14, 224-7, 252, 287-90, 294, 297-9, 312

A Critique of Postcolonial Reason $33,176,218,223$

'Can the Subaltern Speak?' 27, 131, 217-23, 226, 230, 255, 298

'French Feminism in an International Frame' 210

'Subaltern Studies:

Deconstructing

Historiography' 27, 221

'Three Women's Texts and a Critique of Imperialism' $175-8$

Sri Lanka 3, 11, 236

Statute of Westminster (1931) 11

St Kitts 245

strategic essentialism 222, 231, 312

subaltern 130, 155, 218-27, 231, 255, 302

see also Spivak, Gayatri

Chakravorty

'Subaltern Studies' 27, 130, 217-19, 221

Thailand 280

Thieme, John 33, 90, 166-7, 207, 280

'Third World' women 197, 199-200, 207, 209-15, 217, 219, 222, 224-5

Thomas, Nicholas 67 
Tiffin, Chris 44-5

Tiffin, Helen 29-33 see also Empire Writes Back, The

'tricontinent', tricontinentalism 34, 299, 301

Trinidad 11-12, 19, 240-1, 246

Uganda 112, 137

Viswanathan, Gauri 163-5

Walcott, Derek 293

Walder, Dennis 155

Wales 282, 284

Walsh, William 17-18, 280-1

Ware, Vron 201
White, Patrick

Voss 17

White, Paul 242

Williams, Chris 282

Williams, Patrick 9, 33, 58

Wright, Judith 144-5, 149

Wright, Richard 268

Yeats, W. B. 110, 282

Young, Robert J. C. 9, 27, 34-5, 56, 94, 135-6, 220, 286, 299-302

Yuval-Davis, Nira 132, 137, 139, 159

Zephaniah, Benjamin 94, 238

Zimbabwe 14 